\title{
Identificación automática de marcadores argumentativos en discursos políticos
}

\author{
Felipe Ojeda-Cruz ${ }^{1}$, Noé Alejandro Castro-Sánchez ${ }^{1}$, \\ Héctor Jiménez-Salazar ${ }^{2}$ \\ ${ }^{1}$ Departamento de Ciencias de la Computación CENIDET, \\ México \\ ${ }^{2}$ Departamento de Tecnologías de la Información, Unidad Cuajimalpa UAM, \\ México
}

\{felipe.ojeda,ncastro\}@ cenidet.edu.mx, hgimenezs@gmail.com

\begin{abstract}
Resumen. En este artículo se presenta el desarrollo de un método para identificar marcadores argumentativos, al que denominamos "inducción de patrones". Método que a partir de uno o varios patrones, encuentra otros. Los patrones obtenidos se aplicaron a un conjunto de documentos sobre política para identificar marcadores de argumentos. Los resultados obtenidos arrojan una precisión del $92 \%$ y una cobertura del $25 \%$ en la extracción de patrones contextuales de los marcadores.
\end{abstract}

Palabras clave: marcadores argumentativos, Capaldi, Freeling, patrones sintácticos, POS, ete-toolkit, morfosintáctico, identificación de argumentos.

\section{Automatic Identification of Argumentative Markers in Political Speeches}

\begin{abstract}
This article describes the development of a method to identify argumentative markers, which we call "induction patterns". Method from one or more patterns, find others. The patterns obtained are applied to a set of policy documents for to identify markers of arguments. The end result is used to identify arguments with a regular expression. The results show a precision of $92 \%$ and $25 \%$ coverage in the extraction of contextual patterns of markers.
\end{abstract}

Keywords: argumentative markers, Capaldi, Freeling, syntactic patterns, POS, ete-toolkit, morphosyntactic, identification of arguments.

\section{Introducción}

En el marco de la lógica, los argumentos "son conjuntos de razones o de pruebas para apoyar una conclusión"[1], de igual manera los argumentos intentan persuadir y 
convencer a terceras personas de lo que se está proponiendo, es por eso que los argumentos son una parte importante en nuestro lenguaje diario. Argumentamos por muchas razones, por ejemplo, para convencer a nuestro interlocutor sobre algún documental o sobre la importancia de comer saludable, entre muchas otras cosas más.

La naturaleza de los argumentos es muy compleja, hay argumentos implícitos y explícitos, estos últimos hacen uso de palabras claves (marcadores) que facilitan la identificación de los argumentos. Los marcadores consisten en palabras claves que funcionan como una especie de conjunción o de introductores entre proposiciones.

La identificación de argumentos en el área de Inteligencia Artificial es un tema relativamente nuevo, los primeros avances computacionales fueron en el 1st Argument Mining workshop at ACL 2014 y 2st Argument Mining workshop at ACL 2015, en ellos se utilizan diferentes técnicas de procesamiento de lenguaje natural como los ngramas.

Por otro lado, en el presente trabajo se desarrolló un método de inducción de patrones para identificar más marcadores argumentativos y así, permitir identificar más argumentos explicitos; utilizando el analizador sintáctico Freeling[2], ya que es un analizador con una gran precisión morfosintáctica.

Estudiar los argumentos políticos es una tarea interesante porque el problema con los argumentos de cualquier proyecto público es saber fijar qué hay de fundamento y qué hay de excusa. El argumento que se proporciona al público es la reflexión de la toma de decisiones del gobierno y en que medida lo contado es solo la reelaboración de esas ideas, que pueden servir de justificación, para hacer más atractivo o al menos más tolerante a los ciudadanos[3]. Los discursos políticos llevan una estructura de redacción, porque llevan argumentos lógicos, claros y bien definidos; y así son más persuasivos[4], así como también guiar a los ciudadanos a reflexionar como los políticos desean.

\section{Idetificación de argumentos con marcadores}

En este artículo nos centramos únicamente en la identificación de argumentos que contengan marcadores de razón y de conclusión, es por ello que se tomó la tarea de investigar cuáles son los marcadores y cómo identificarlos. En [5] se menciona el uso de palabras claves de premisas y conclusión para identificar el argumento en un discurso general. De igual forma plasma algunas ideas y reglas que facilitan esta identificación. Algunas palabras que introducen conclusión son: "como resultado", "de ahí que", "en consecuencia", "se desprende que", "llegamos a la conclusión", "por consiguiente" y sinónimos. Algunas palabras que introducen razones son: "puesto que", "ya que", "como", "en tanto que", "dado que", "por cuanto", "viendo que" y sinónimos.

Ya teniendo un conjunto de marcadores se analizaron algunos párrafos que son argumentos. Cabe destacar que el primer paso para identificar argumentos es identificar la proposición que funciona como conclusión[1], y si se encuentra, es que el párrafo es un argumento y se compone de dos partes (premisas y conclusión).

Sin embargo, un párrafo puede que tenga más de una conclusión, y que dicha conclusión funcione como premisa, es decir, toda proposición puede ser tanto una premisa como una conclusión; a esta serie de argumentos interrelacionados se le conoce como sorites[5]. 
Para resolver el problema de identificar automáticamente argumentos por marcadores, así como también los sorites; se tomó la decisión de implementar expresiones regulares (regex) para analizar todo el discurso.

La expresión regular que se desarrolló (Fig. 1), consiste en dos grandes partes, que se explicarán a detalle en el siguiente apartado. La expresión regular puede identificar las premisas y conclusiones sin importar su posición en el párrafo, de igual forma no se identifican por su contenido. Esto es importante ya que, por la naturaleza de los argumentos, la conclusión se puede dar en cualquier parte del párrafo.

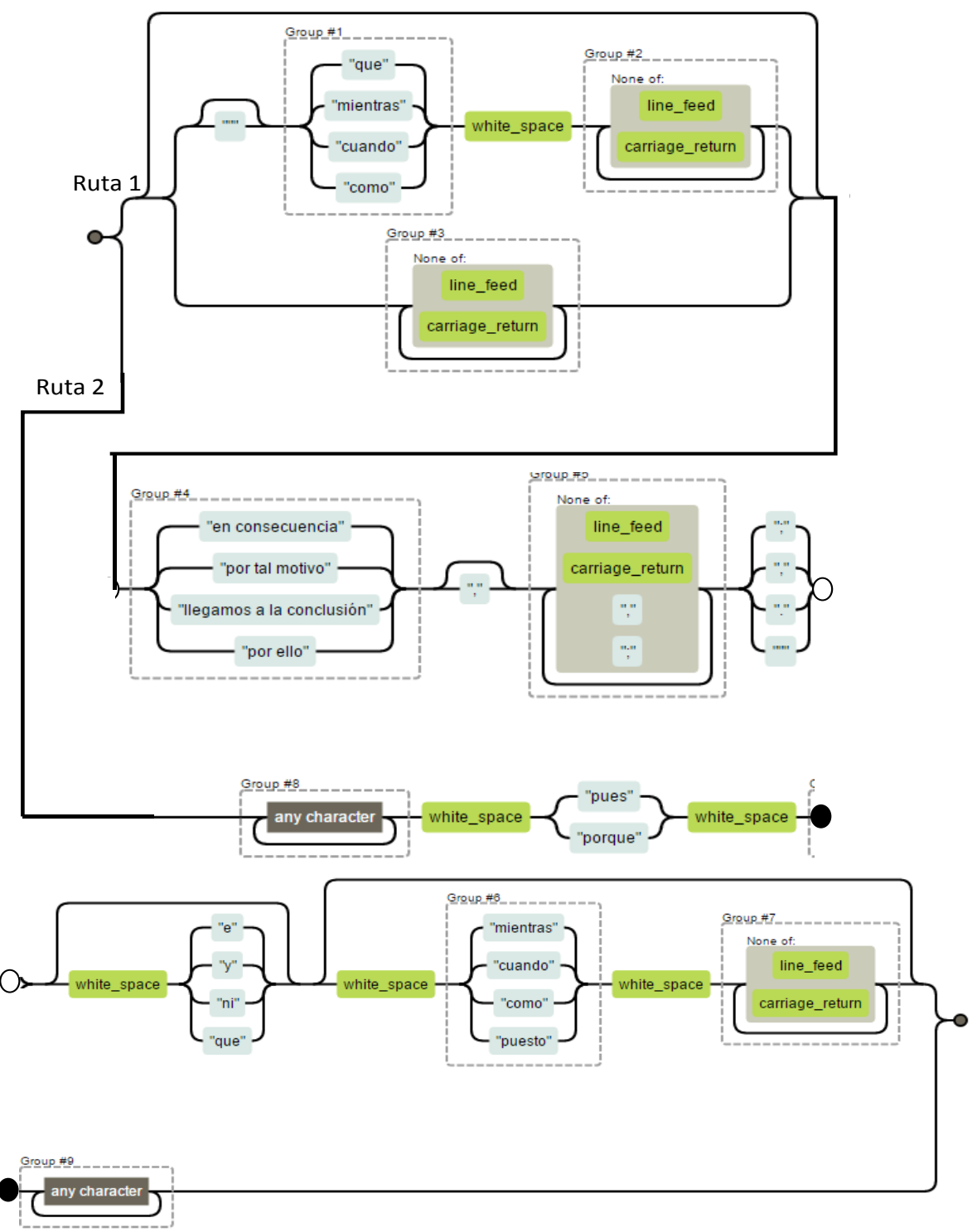

Fig. 1. Diagrama de ferrocarril del regex para identificar argumentos[6]. 


\subsection{Explicación del diagrama}

La expresión regular empieza con dos rutas, la ruta 1 es la primera parte y se divide en tres sub-partes más. La primera sub-parte de la expresión regular (grupo del 1 al 3) identifica el conjunto de premisas pero esta identificación es opcional. La segunda parte (grupo del 4 al 5) es la conclusión y no es opcional, de esta forma se pretende encontrar un argumento sobre cualquier de las siguientes estructuras, premisa-conclusión, conclusión-premisa o premisa-conclusión-premisa. Por último tenemos la tercera subparte que consiste en identificar premisas, de igual manera opcional con la diferencia que implica, por ejemplo, no iniciar con algunos caracteres.

Aparte de los marcadores de conclusión, existen otras palabras que permiten identificar premisas y conclusiones al mismo tiempo; por mencionar dos casos, "pues" y "porque". Lo que va después de estas palabras, indica la existencia de una o más premisas, y lo que va antes indica la existencia de conclusión. Por tal motivo, como se aprecia en la figura 1, existen al inicio dos caminos por recorrer; iniciando en el grupo \#1 o iniciando desde el grupo \#8 (ruta 2).

La expresión regular se divide en nueve grupos, cada uno captura diferentes partes del argumento:

- Grupo \#1: Captura el introductor de premisa.

- Grupo \#2: Captura la proposición-premisa.

- Grupo \#3: Captura la proposición-premisa pero que no cuenta con un introductor de premisa.

- Grupo \#4: Captura el introductor de conclusión.

- Grupo \#5: Captura la proposición-conclusión.

- Grupo \#6: Captura el introductor de premisa que se encuentra después de una conclusión.

- Grupo \#7: Captura las proposiciones-premisa que se encuentran después de un introductor de premisa.

- Grupo \#8: Captura la proposición-conclusión determinado por los marcadores "pues" o "porque".

- Grupo \#9: Captura la proposición-premisas determinado por los marcadores "pues" o "porque".

No todos los grupos capturarán al mismo tiempo, o se activan los grupos \#1-7 o los grupos \#8-9, en los casos del grupo \#1-7 se activarán 3 grupos y en los grupos \#8-9, siempre se activarán los dos.

Una vez que se identifique el cuerpo del argumento, se aplica de nuevo la expresión regular para identificar si existen otros argumentos dentro del argumento principal y así sucesivamente, hasta que el algoritmo no encuentra ningún introductor de conclusión.

Por otra parte, la consideración de signos de puntuación y/o conjunciones en los argumentos proporciona ciertas pautas que indican proposiciones. Capaldi [5] menciona la importancia de su uso en argumentos por ser utilizados en la mayoría de los casos, por esa razón se consideraron los signos de puntuación en la expresión regular (véase entre los grupos \#5 y \#6). 


\subsection{Identificar argumentos interrelacionados}

En la mayoría de los casos un argumento está dividido en premisas y conclusión, sin embargo la conclusión puede funcionar como premisa para otro argumento, a esto se le conoce como argumentos interrelacionados o sorites. En teoría, para identificar todos los argumentos que puede haber en un fragmento textual como un párrafo, se aplicaría varias veces la expresión regular, para encontrar los demás argumentos. La razón de aplicarlo de nuevo es que en su defecto encuentran la primera coincidencia y continúan hasta donde finalizó la coincidencia, por lo que no hacen búsqueda superpuesta. Las expresiones regulares tienen la opción de realizar búsquedas superpuestas, sin embargo aplicar esta opción puede desencadenar retroceso catastrófico, es un concepto conocido cuando su motor regresa a un estado anterior para analizar los otros posibles casos en la búsqueda del texto[7].

Por ejemplo, dado el siguiente argumento extraído del sitio www.gob.mx:

"El Programa Nacional de Sustitución de Lámparas Incandescentes por Ahorradoras de Energía busca proteger la economía de las familias más necesitadas; así los hogares reducirán su consumo, y por lo tanto, pagarán menos.”

Al utilizar el método de la expresión regular, identifica dos partes del texto. Lo que está antes de "por lo tanto" son las premisas y lo que está después es la conclusión. Después se tiene como entrada el fragmento de las premisas, la expresión regular identificará de igual forma dos partes; lo que está antes del marcador "así" es la premisa y lo que está después es la conclusión hasta llegar a consumo. De esta forma, se puede identificar todos los argumentos que puede haber en un párrafo.

\subsection{Incremento de marcadores}

El alcance de la identificación de argumentos con una expresión regular está limitada por el número de marcadores. Así que el siguiente paso que se hizo, fue buscar más sinónimos de los marcadores llegando a una lista más extensa. La tabla 1 indica con asteriscos algunos marcadores que menciona Capaldi, aquellos que no lo tienen son algunos de los encontrados por los recursos sensagent, interglot, thefreedictionary y RAE; como también de los autores Calsamiglia y Tuson[8].

Tabla 1. Muestra de marcadores argumentativos encontrados por algunos recursos.

\begin{tabular}{lll}
\hline Marcadores de conclusión & & Marcadores de premisas \\
\hline por consiguiente & $*$ & al igual que \\
se desprende que & $*$ & como \\
como resultado & $*$ & igual que \\
luego & & tan \\
llegamos a la conclusión & $*$ & en tanto que \\
entonces & & en el supuesto de que \\
así pues & & en la medida en que \\
\hline
\end{tabular}


El número de marcadores de premisas adicionales son 28 y el de conclusiones son 30. Sin embargo es posible que dependiendo del dominio del tema o el tipo de discurso (oral o escrito) se utilicen otros marcadores argumentativos. Ante este hecho, se desarrolló el método para extraer más marcadores, como se verá en el siguiente capítulo.

\section{Método de inducción de patrones}

El método de inducción se basa en la siguiente consideración. Sea T un texto con etiquetas POS (part of speech) que contiene un marcador de Capaldi, M. Considérese el contexto izquierdo C_i y derecho C_d de M en T; es decir, la cadena C_iMC_d ocurre en T. Si para otro término $M$ ' se cumple que $C_{-} i M^{\prime} C_{-} d$ ocurre en $T$, diremos que $M$ ' satisface el contexto de $\mathrm{M}$ y, por lo tanto, puede ser un marcador equivalente a M. Este método tomó como base las ideas presentadas por Marti Hearst en la identificación de hipónimos [9]. Además puede aplicarse este enfoque de inducción en la identificación de definiciones[10].

El método desarrollado consta de dos fases, el método para la extracción automática de patrones contextuales mediante el análisis morfosintáctico, y el método para la búsqueda extendida de expresiones regulares con patrones morfosintácticos.

El método de extracción de patrones requiere como parámetros un conjunto de textos y, y el marcador o marcadores que se quieren analizar. Después del análisis automático se genera una tabla con estadísticas que muestra un conjunto de etiquetas chunking y Eagle, cada tipo de etiqueta se acompaña de la cantidad de veces que apareció en los contextos de la palabra analizada y también se acompaña del porcentaje con respecto al total de textos analizados.

El método siguiente, requiere como entrada una expresión regular, que a su vez implica contener las palabras claves y la sintaxis de estos. Asimismo la expresión regular puede estar conformada de etiquetas Eagle o Chunk, que se identifica del texto o caracteres especiales, porque cada etiqueta tiene al final guion bajo “_". El resultado es una expresión regular con las etiquetas sustituidas por texto.

\subsection{Método de extracción automática de patrones contextuales mediante el análisis morfosintáctico}

Freeling posee varios análisis; análisis morfológico, etiquetado POS, análisis de dependencia, análisis sintáctico de poca profundidad, y análisis sintáctico completo. Este último fue el utilizado para identificar los patrones que rodean a los marcadores.

El análisis sintáctico además de contener análisis morfológico contiene las relaciones entre palabras y oraciones, lo que incrementa la posibilidad de extraer patrones más precisos, hasta llegar a un grado de patrones a nivel de sintagmas u oraciones. Estas relaciones entre palabras, Freeling lo llama Chunking Tags (Fragmentos de etiquetas). Por mencionar algunas de las decenas que existen, son: a-fp (Adjetivo, Adjetivo femenino plural), coord. (Conjunción, Conjunción de coordinación), grup-sp (Preposición, fragmento preposición), entre otras[11].

En un análisis sintáctico completo que realiza Freeling, se puede observar en la Fig. 2. 
Por ejemplo, se tomó un fragmento textual del discurso "Estado mayor presidencial" del sitio www.gob.mx en el que se menciona lo siguiente[12]:

"El Estado Mayor Presidencial (EMP) es un órgano técnico militar que tiene como misión fundamental proteger al Presidente de la República. Esta responsabilidad es de la más alta importancia para el País, 'pues' implica el resguardo de la representación del Estado Mexicano y de la República, 'por consiguiente' de la estabilidad, de la gobernabilidad y de la seguridad nacional".

En el párrafo citado se observa dos marcadores "pues" y "por consiguiente". Obsérvese en la figura 2 el nodo raíz con la etiqueta "RG" que representa el introductor de conclusión "por consiguiente", el contexto inmediato a nivel POS (Etiquetas Eagle), son las etiquetas izquierdo "Fc" y derecho "SPS00" y a nivel sintáctico (Etiquetas Chunk), el contexto izquierdo son "Fc" y derecho "sp-de". Nótese que la etiqueta "spde" representa el fragmento textual "de la estabilidad".

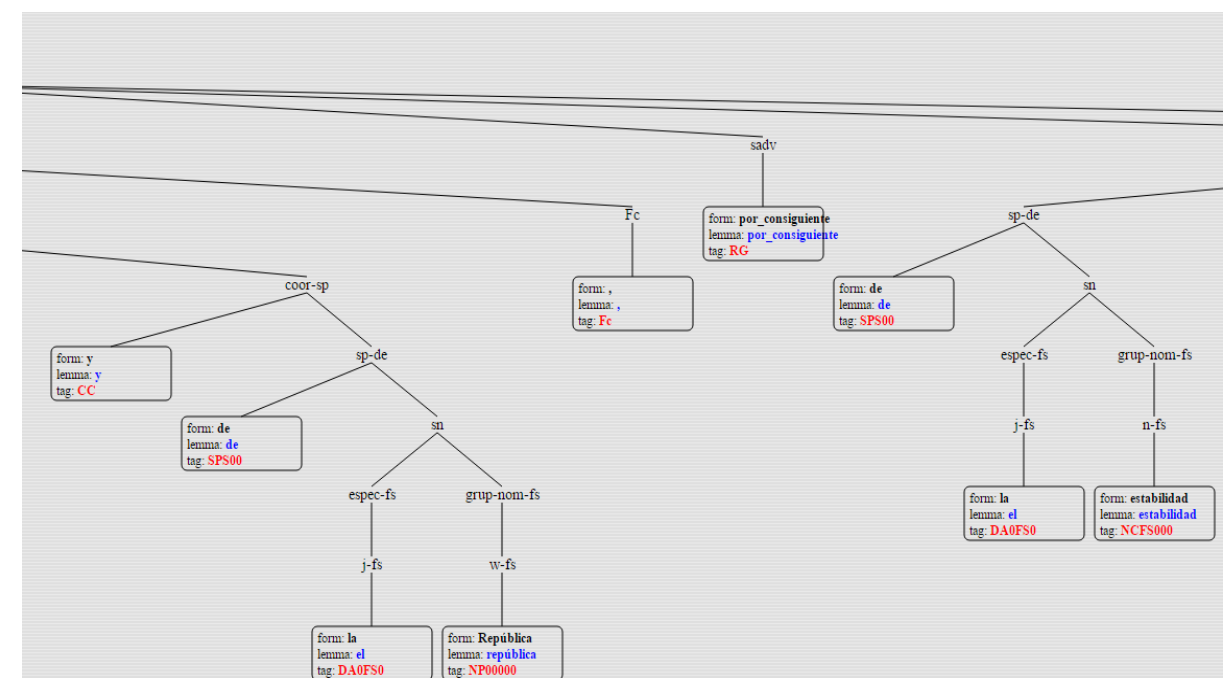

Fig. 2. Representación parcial de un árbol sintáctico del párrafo citado.

Sin embargo, en la mayoría de las situaciones el contexto izquierdo de una palabra puede tener más de una etiqueta Chunk como sucede con la ",". Analizando el lado izquierdo tenemos que "w-fs, grup-nom-fs, sn, sp-de, coor-sp" son el contexto izquierdo de primer nivel; esto se sabe, porque si traducimos las etiquetas a texto tenemos las siguientes combinaciones:

- Coor-sp: y de la República “,” por consiguiente...

- Sp-de: de la República "," por consiguiente...

- Sn: la República “,” por consiguiente...

- Grup-nom-fs y w-fs: Repúblia “,” por consiguiente...

Los niveles contextuales consisten, a partir del nodo padre en común con la palabra a ser analizada; si se analizara el segundo nivel izquierdo, empezaría por la etiqueta "DA0FS0" hasta recorrer el árbol llegando al nodo padre, sin considerar este último.

Sobre este enfoque se desarrolló un método automático qué, dada la forma de la palabra, el lema o la etiqueta Eagle, y el árbol sintáctico de Freeling; proporciona como 
resultado una tabla de estadísticas, donde se observan los patrones contextuales de las palabras analizadas.

Por otra parte, con el apoyo de ETE Toolkit[13] se desarrolló el método de extracción automática de patrones. ETE Toolkit es un marco en python que permite el análisis y visualización de árboles. La manipulación de esta herramienta con los árboles hace más fácil interactuar con los árboles de Freeling. ETE Toolkit se desarrolló en un principio para la filogenómica, por lo que utilizarlo en árboles de Freeling tendría mucho potencial, por consiguiente podría utilizarse para muchos análisis más.

\subsection{Método para la búsqueda extendida con expresiones regulares de patrones morfosintácticos}

Las expresiones regulares, también conocidas como regex, regexp o regexen, son patrones textuales que realizan búsquedas a nivel de sintaxis[14].

Sin embargo en este trabajo se extendió su funcionalidad, a tal grado de poder realizar búsquedas a nivel morfosintáctico. Para llegar a esa funcionalidad se realiza un pre-procesamiento de análisis morfosintáctico.

Se procede de la siguiente manera:

1. Se proporciona manualmente un texto de entrada, y se analiza automáticamente el texto extrayendo el contexto de las palabras analizadas.

2. De manera automática, se obtiene un árbol con la API de Freling y se codifica al formato Newick para su posterior utilización con ETE.

3. Se eliminan etiquetas redundantes automáticamente.

4. Se crea automáticamente una expresión regular con la posibilidad de respetar la posición de las etiquetas Eagle o Chunk.

5. Se proporciona manualmente otro texto a analizar para encontrar marcadores argumentativos.

6. Se busca la representación textual de las etiquetas en el texto proporcionado de manera automática.

7. Se sustituye automáticamente la representación textual a cada una de las etiquetas que están en la expresión regular.

En el paso 6 se aplica la expresión regular al segundo texto proporcionado, para encontrar los marcadores que respeten el patrón extraído del primer texto.

El paso 4 puede omitirse, entonces se debe colocar una expresión regular propia o la que genera el sistema, por ejemplo considerando las etiquetas con la mayor probabilidad, esto lo proporciona el método anterior (sección 3.1).

\section{Experimentos y resultados}

Dado que Freeling maneja una precisión aproximada del 97\%[15], se eligió para realizar el etiquetado POS. De igual manera, cabe señalar que la precisión que arrojan los métodos desarrollados dependerá en parte de Freeling.

Para analizar la efectividad de los métodos, se realizó una consulta manual de 200 discursos de la presidencia de la República y secretarías federales, de los cuales 29 contienen marcadores conocidos y de ellos se extrajeron manualmente varios 
argumentos con diferentes marcadores de conclusión sugeridos por Capaldi. En la siguiente tabla (tabla 2) se indican los marcadores extraídos:

Tabla 2. Marcadores argumentativos identificados en discursos políticos.

\begin{tabular}{lcc}
\hline & Marc. Arg. & No Marc. Arg. \\
\hline Como resultado & 3 & 3 \\
De ahí & 5 & 1 \\
En consecuencia & 9 & \\
Implica & 1 & \\
Se llegó & 1 & \\
Por consiguiente & 1 & 2 \\
Por lo tanto & 17 & 37 \\
Así & 9 & 1 \\
Por ello & 6 & 44 \\
Total & 52 & \\
\hline
\end{tabular}

La intensión es generar expresiones regulares que incluyan los patrones antes indicados. De la tabla anterior se observa que los más utilizados son "por lo tanto", "en consecuencia" y "así'. Dado que este último presenta mucha ambigüedad porque puede funcionar como adverbio, conjunción, interjección, entre otras; se decidió tomar los dos primeros para hacer la generación automática de expresiones regulares. En total se logró generar automáticamente 16 expresiones regulares (16 pruebas), de las cuales fueron dos las que lograron identificar la mayor cantidad de palabras que sí cumplen la función de marcadores.

El siguiente fragmento es tomado de una expresión regular. Fue generada por el sistema, tomando como datos de entrada 20 argumentos con el marcador "por lo tanto" y 5 de "en consecuencia" obtenidos de distintos discursos:

\s((?:RG_)|(?:SPS00_)\s[\wáéíóú]+(?:\s(?:[\wáéíóú]+))?|(?:CS_)(?:\s(?:[|wáéíóú]+))?|( ?:P00CN000_)\s[|wáéíóú]+(?:\s(?:[|wáéíoú]+))?)((?:Fc_) (?:P00C_) (?:VMIP_)

$\left(?: \mathrm{DAOF}_{-}\right)\left|\left(?: \mathrm{Fc}_{-}\right)\left(?: \mathrm{P} 00 \mathrm{C}_{-}\right)(?: \mathrm{VMIF})\left(?: \mathrm{VMN} \mathrm{O}_{-}\right)\right|\left(?: \mathrm{DA} 0 \mathrm{~F}_{-}\right)\left(?: \mathrm{NCFS}_{-}\right)$

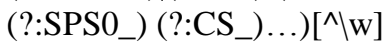

La expresión regular mostrada contiene etiquetas POS truncadas para considerar una mayor posibilidad de encuentros, ya que además de que se extrajeron únicamente los patrones POS también se consideró la posición, por ejemplo: el contexto de un argumento en particular con el marcador "por lo tanto" contiene las etiquetas PP1,VMI,Fc; y se está respetando la posición de estos. Es decir, primero tiene que estar la etiqueta PP1, seguida de la etiqueta VMI y posteriormente la etiqueta Fc, así también con los demás argumentos analizados.

Un ejemplo de la expresión regular mostrada es la identificación del marcador de conclusión "de ahí" del siguiente argumento: "El diagnóstico de la pobreza y el diálogo de la pobreza se vuelve cada vez más complicado y de ahí la importancia en que permanentemente midamos para ver cómo estamos evolucionando"[16]. El contexto 
derecho del marcador "de ahí" es: "la importancia en que" que en términos morfosintácticos es: DA0F $\rightarrow$ la, NCFS $\rightarrow$ importancia, SPS $0 \rightarrow$ en, CS $\rightarrow$ que. Así la expresión regular identificó un nuevo marcador tomando como base los marcadores "por lo tanto" y "en consecuencia"

Tabla 3. Resultados de los marcadores argumentativos encontrados con el método de inducción.

\begin{tabular}{lcc}
\hline Inducción de patrones (Reglas-aplicadas) & Precisión & Cobertura \\
\hline Experimento 1 & $56 \%$ & $25 \%$ \\
Experimento 2 & $92 \%$ & $25 \%$ \\
\hline
\end{tabular}

Se realizarón algunos experimentos, de las cuales dos de ellas fueron las más precisas, los resultados se pueden observar en la tabla 3. El experimento uno tiene las siguientes características: posición de los patrones, que se refiere al orden de las etiquetas POS; contexto izquiero y derecho (3 niveles), se refiere a que sólo se tomaron 3 palabras a la izquierda y derecha; patrones de "por lo tanto" y "en consecuencia", son los marcadores analizados; y truncado a 2 , se refiere a que no se consideró toda la anotación morfosintáctica. El experimento dos, sólo se consideró el contexto derecho (4 niveles); es decir cuatro palabras a la derecha; truncado a cuatro, es decir los cuatro primero caracteres de las etiquetas; posición de los patrones, el órden de las etiquetas fueron tomados en cuenta; tipo de marcador, se refiere al tipo de información morfológica, por ejemplo, marcadores de tipo adverbio; y patrones de "por lo tanto" y "en consencuencia", que son los marcadores analizados.

\section{Conclusiones y trabajos futuros}

Respecto a los resultados que arrojaron baja precisión, posiblemente se deba a que no se consideraron más argumentos de prueba para extraer sus patrones y de igual forma, es posible que hiciera falta analizar otros tipos de marcadores para luego extraer sus patrones contextuales. Sin embargo, debido a que no se encontró ningún corpus de argumentación en español se tuvo que identificar manualmente argumentos de varios discursos políticos, y desde luego la dificultad que existe al identificar argumentos con algunos marcadores ambiguos hizo el trabajo más tedioso.

Por otro lado, sobre la explicación anterior, se plantea la posibilidad de aplicar las ideas propuestas para tener una variedad más rica de patrones, para que así, encuentre otro tipo de marcadores que cumplan con los requisitos del patrón POS. De tal forma, que aplicando este principio se incremente la precisión y cobertura.

Los métodos desarrollados serán optimizados para aplicarse en diferentes escenarios. Asimismo, la posibilidad de eliminar redundancia de información al encontrar los patrones y las palabras sustituidas.

Además, se realizarán más pruebas que nos permitan identificar más marcadores de conclusión y empezar a realizar pruebas con las premisas.

Los métodos desarrollados pueden ser utilizados para contextos de diferentes palabras. Por ejemplo, es posible ser utilizado para encontrar palabras claves que indiquen definiciones[10]. 
De igual forma, para la expresión regular mencionada al principio (Figura 1), se plantea la posibilidad de evitar ambigüedad en los argumentos sobre determinados marcadores, por ejemplo "así'. Según el diccionario de la RAE, la palabra "así" puede tener muchos significados dependiendo de su contexto, por ejemplo: "con sueldos así no se puede vivir", en esta oración "así" está funcionando como adjetivo, y sus sinónimos pueden ser las palabras "semejante o similar"[17], claramente se aprecia que esta palabra no actúa como marcador argumentativo. Por ello, los marcadores ambiguos pueden ser descartados de la identificación teniendo mayor cantidad de ejemplos para diferenciarlos de los que efectivamente son marcadores de argumentos.

Agradecimiento. Los autores agradecen al Consejo Nacional de Ciencia y Tecnología por el apoyo otorgado al presente trabajo.

\section{Referencias}

1. Weston, A.: Las claves de la argumentación. 4th ed. Grupo Planeta (2011)

2. Padró, L.: Analizadores multilingües en freeling. Linguamática, Vol. 3, No. 2, pp. 13-20 (2012)

3. Ferrari, E.: Los argumentos políticos, [Online]. Available: http://www.elnortedecastilla. es/20081219/articulos_opinion/argumentos-politicos-20081219.html. [Accessed: 04-May2016] (2008)

4. Educación: Cómo hacer un discurso perfecto, según el escritor que se los redacta a Obama. [Online]. Available: http://www.elconfidencial.com/alma-corazon-vida/2015-0115/6-consejos-para-elaborar-un-discurso-convincente-segun-el-asesor-de-obama_621098/. [Accessed: 04-May-2016].

5. Capaldi, N.: Como ganar una discusión. Tercera (2011)

6. Avallone, J.: Regexper [Online]. Available: www.regexper.com.

7. Microsoft: Retroceso en expresiones regulares. [Online]. Available: https://msdn.microsoft.com/es-es/library/dsy130b4(v=vs.110).aspx\#controlling backtracking [Accessed: 20-Mar-2016]

8. Calsamiglia, H., Tusón, A.: Las cosas del decir: Manual del análisis del discurso (1999)

9. Hearst, M.A.: Automatic acquisition of hyponyms from large text corpora. Proceedings of the 14th conference on Computational linguistics, Vol, 2, pp. 539-545 (1992)

10. Alarcón, R., Sierra, G., Bach, C.: Developing a Definitional Knowledge Extraction System. Proc. 3rd Lang. Technol. Conf., pp. 374-379 (2007)

11. Lloberes M., Padro, L.: Freeling shallow parser: Chunking tags for spanish [Online]. Available: http://devel.cpl.upc.edu/freeling/svn/trunk/doc/grammars/esCHUNKtags. [Accessed: 03-Mar-2016]

12. P. de la República: Estado Mayor Presidencial | Presidencia de la República | Gobierno | gob.mx. [Online]. Available: http://www.gob.mx/presidencia/acciones-yprogramas/estado-mayor-presidencial-14579. [Accessed: 03-Mar-2016] (2015)

13. Huerta-Cepas, J., Serra, F., Bork,P.: ETE 3: Reconstruction, analysis and visualization of phylogenomic data. Mol. Biol. Evol., p. msw046 (2016)

14. López, F., Romero, V.: Mastering Python Regular Expressions. Packt Publishing Ltd, (2014)

15. Padró, L., Collado, M., Reese, S., Lloberes, M., Castellón, I.: FreeLing 2.1: Five Years of Open-Source Language Processing Tools(2010)

16. S. de D. Social: Mensaje del secretario Meade en la reunión del Consejo Directivo de la Cámara Nacional de la Industria de la Transformación (Canacintra). [Online]. Available: http://www.gob.mx/sedesol/prensa/mensaje-del-secretario-meade-en-la-reunion-del- 
Felipe Ojeda-Cruz, Noé Alejandro Castro-Sánchez, Héctor Jiménez-Salazar

consejo-directivo-de-la-camara-nacional-de-la-industria-de-la-transformacion-canacintra.

[Accessed: 06-May-2016] (2015)

17. DLE: así - Diccionario de la lengua española - Edición del Tricentenario. [Online].

Available: http://dle.rae.es/?id=3zT41MT. [Accessed: 14-Mar-2016] 